\title{
Autoimagen, Autoconcepto y Autoestima, Perspectivas Emocionales para el Contexto Escolar
}

\author{
Rafael Enrique Buitrago Bonilla ${ }^{1}$ \\ Nancy Yanneth Sáenz Salcedo ${ }^{3}$
}

\footnotetext{
1. Universidad Pedagógica y Tecnológica de Colombia lafaremus@gmail.com

2.Institución Educativa Juan José Reyes Patria
}

Como citar: Buitrago, R., \& Sáenz, N. (2021). Autoimagen, Autoconcepto y Autoestima, Perspectivas Emocionales para el Contexto Escolar. Educación y Ciencia, (24), e 12759. https://doi.org/10.19053/0120-7105. eyc.2021.25.e12759

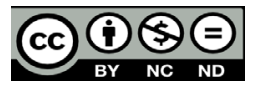

\section{Resumen}

El artículo aborda algunos aspectos del ámbito intrapersonal en el contexto escolar, para ello, se plantean tres apartados: Inteligencia Emocional [IE] en el contexto escolar, en el que se hace explicita la importancia de la IE para las personas, en este caso, para el profesorado, el alumnado y las demás personas que intervienen y hacen parte de los procesos educativos, además, se resalta que la escuela es fundamental para su expresión, interacción y desarrollo; Autoimagen, Autoconcepto y Autoestima, aspectos fundamentales para el aprendizaje, plantea elementos importantes respecto a la incidencia de estas tres dimensiones para los procesos de aprendizaje y desarrollo del pensamiento, los cuales se fundamentan, en gran medida, en las relaciones interpersonales; Autoimagen, Autoconcepto y Autoestima del profesorado, dedicado a resaltar la relevancia de este ámbito intrapersonal para el bienestar personal, laboral y social del profesorado y para el éxito escolar. Por último se concluye con una reflexión final, en donde se señala la relevancia de abordar el desarrollo de habilidades socioemocionales tanto en la escuela como en la formación del profesorado.

Palabras Clave: inteligencia emocional, autoimagen, autoconcepto, autoestima, profesorado.

Recibido: 25/04/2021 | Revisado: 03/05/2021

Aprobado: 17/06/2021 | Publicado: 19/10/2021 


\title{
Self-Image, Self-Concept and Self-Esteem, Emotional Perspectives for the School Context
}

\begin{abstract}
The paper presents some aspects of the intrapersonal field in the school context, from three sections: Emotional Intelligence in the School Context, which shows the importance of EI for people, in this case, for teachers, students and the other people who intervene in the educational processes, in addition, it is emphasized that the school is fundamental for its expression, interaction and development; Self-image, Self-concept and Self-esteem, fundamental aspects for learning, where fundamental aspects are raised regarding the incidence of these three dimensions for the processes of learning and development of thought, which are privileged with interpersonal relationships; Self-image, Self-concept and Self-esteem of teachers, dedicated to highlighting the relevance of this intrapersonal area for the personal, work and social well-being of teachers and for school success; Finally, it concludes with a final Reflection, where the relevance of addressing the development of socio-emotional skills is pointed out both in school and in teacher training.
\end{abstract}

Keywords: emotional intelligence, self-image, self-concept, self-esteem, teachers.

\section{Inteligencia Emocional en el Contexto Escolar}

Adolphs (2005) señala que la emoción es "un cambio fásico, concertado, generalmente adaptativo, de múltiples sistemas fisiológicos (con componentes somáticos y neurales) en respuesta a la evaluación de un estímulo" (pp. 13-14). Por consiguiente, se puede afirmar que las emociones hacen parte de la vida y cotidianidad de las personas, de sus vínculos, relaciones y contextos. De igual manera, se puede señalar que, aunque estas no son ni buenas ni malas y su incidencia está asociada a cada situación y contexto, el impacto que generan en las personas permite definirlas como positivas o negativas (Mestre et al., 2017). Es relevante señalar que cada lóbulo cerebral está asociado a cada tipo de emoción, siendo el izquierdo el que se relaciona con las positivas y el derecho con las negativas (Davidson et al., 2000). Aunque las emociones tienen una función particular y por lo tanto se deben saber gestionar (Buitrago, 2012), las acciones, conductas y comportamientos pueden generar malestar o no ser pertinentes para la persona o para los demás (Greenberg, 2003), por esta razón, es importante aprender a identificar en qué momentos se es presa y se está sometido a una emoción determinada (Maganto \& Maganto, 2010).

Las emociones pueden tomar características relevantes o ser dañinas e inservibles, por esta razón es que cada experiencia específica da el tinte de positivo o negativo, 
pero es preciso comentar que, al acercarse a experiencias emocionales intensas se suele perder el control con facilidad (Dalai-Lama \& Ekman, 2009; Ekman, 2003). En esta dirección, experimentar emociones positivas está asociado a estados de bienestar, mientras que las negativas pueden llegar a generar afectación individual y colectiva debido a que limitan el pensamiento (Dalai-Lama \& Ekman, 2003; Maganto \& Maganto, 2010). Desde esta perspectiva, es evidente que las emociones inciden en la interioridad de las personas, en sus vínculos, y en los contextos familiares y sociales. De manera desafortunada, en muchas ocasiones las emociones se reprimen, se excluyen o no se visibilizan, incidiendo por lo tanto en la autoimagen, el autoconcepto y la autoestima (Extremera \& Fernández-Berrocal, 2015), es por esto que la escuela y el profesorado tienen una enorme posibilidad de aportar al desarrollo intrapersonal y al bienestar propio y del alumnado.

Por todo ello, la Inteligencia Emocional [IE] es un aspecto relevante que debe estar presente en los contextos escolares, porque incide en todas las personas, en el profesorado, quien debe contar con una serie de habilidades y recursos emocionales que le posibiliten el éxito laboral, la felicidad, la estabilidad, el bienestar y la consolidación de su identidad profesional docente (Buitrago-Bonilla \& CárdenasSoler, 2017; Zembylas, 2003); en el alumnado, porque mientras se desarrolla, configura y asume posturas críticas en la vida, va aprendiendo a reconocer las expresiones y estados emocionales y a mejorar sus procesos comunicativos (Porras et al., 2020), a ser empático y altruista, a manejar las dificultades y obstáculos, además de potenciar el aprendizaje, los pensamientos y las ideas, pero, ante todo, a configurar vínculos y relaciones interpersonales positivas; en los directivos docentes, para ejercer liderazgo, manejar el estrés, la presión y la incertidumbre, tomar decisiones soportadas y trabajar desde una perspectiva de bienestar colectivo y, desde luego, al igual que para todas las personas, fortalecer su interioridad y bienestar individual y colectivo; en los padres de familia, porque además de su interioridad, la cual en muchas ocasiones es dejada de lado por las circunstancias, compromisos y retos familiares, permite desarrollar habilidades que aporten a las relaciones familiares y en particular con sus hijos, propiciando mejores ambientes y vínculos de bienestar; por último, en el personal de apoyo, quienes en muchos momentos no logran sentirse reconocidos y valorados, pero que igual requieren desarrollar habilidades para la adaptabilidad, la empatía, el manejo del estrés y la gestión de sus emociones.

Además de ello, la IE aporta al clima laboral y organizacional, a los procesos administrativos y académicos, a la intuición y sensibilidad social, al respeto a la diferencia y al trabajo colectivo y en red. Por todo ello, Pérez y Filella (2019) plantean que debe integrarse la educación emocional como una innovación psicopedagógica que se enfoque en la formación para la vida que tiene que realizar la escuela, además, se debe apostar por desarrollar procesos de educación emocional que propicien ambientes de aprendizaje cálidos, motivantes y positivos (Agulló et al., 2011; Brackett et al., 2004; Buitrago et al., 2019; Cherniss et al., 2006; Eren et al., 2009; Herrera et al., 
2017; Herrera, Buitrago, Lorenzo, \& Badena, 2015; Herrera, Buitrago, Lorenzo, \& Perandones, 2015; Gil-Olarte et al., 2006; Greenberg et al., 2003), así como un clima emocional y social de aula positivo (Barrientos et al., 2019; Hubel et al., 2020). Por su parte, Extremera et al. (2019), Engels et al. (2021), así como Amador y Treglown (2020) enfatizan en la importancia de trabajar en torno a la relación de la IE y el engagement, mientras que Palomera et al. (2019) apuestan por la formación en valores y competencias personales del profesorado, las cuales se relacionan con habilidades de autogestión, liderazgo, trabajo colaborativo y en equipo, y comunicación asertiva (Cańas \& Hernández, 2019). Sumado a ello, Rendón (2019) plantea que se deben abordar los estilos y maneras de enseñanza del profesorado desde el crisol de las emociones, así como de la construcción del concepto y la experiencia de paz (Gutiérrez-Torres \& Buitrago-Velandia, 2019).

\section{Autoimagen, Autoconcepto y Autoestima, Aspectos Funda- mentales para el Aprendizaje}

La escuela es un lugar privilegiado donde el aprendizaje es una actividad vital, permanente y continua, que se soporta, sobre todo, en las interacciones con otras personas, con materiales, con pensamientos y con ideas. Por esta razón, Masschelein y Simons (2014) se refieren a la escuela como un tiempo-espacio en el que es posible lograr una suspensión para alejarse de lo cotidiano, sin dejar de pensarlo y analizarlo. Cuando la escuela logra esa especie de interrupción, se da paso a la posibilidad de reconocerse como estudiante, docente y ser humano, así como de escoger qué tipo de conocimientos se resaltan e interiorizan, aspecto que fortalece el rendimiento académico, el éxito y bienestar y, de alguna manera, la formación integral (ArizaHernández, 2017). Por lo tanto, los ambientes, dinámicas y espacios que genera la escuela son fundamentales y difíciles de configurar en otros contextos o circunstancias.

A esto se suma que, si bien es cierto que la escuela posibilita el reconocimiento y valoración de sí mismo, este proceso requiere estructurarse y trabajarse desde los diferentes saberes e interacciones. Por esta razón, el maestro tiene una responsabilidad esencial que implica, además de su desempeño profesional y su adecuada formación, el desarrollo de una serie de habilidades socioemocionales que le permitan disfrutar del placer de la enseñanza y apoyar la configuración individual de cada alumno. Por ello, la autoimagen que cada persona construye tanto de su físico como de los aspectos que se han interiorizado respecto a sí misma, a partir de las propias vivencias $\mathrm{y}$ de las apreciaciones de otros, se convierte en un factor relevante y fundamental para las interacciones y el aprendizaje (Acòn, et al., 2019). Hill (2013) plantea que la autoimagen se integra por la percepción física, los roles sociales y los rasgos personales, no obstante, es común que esta representación sea desacertada o sesgada y por lo tanto, incida de manera negativa en lo que las personas pueden o no llegar a hacer. Al respecto, Gómez-Mármol et al. (2017) señalan que es frecuente que un 
número importante de jóvenes no estén conformes con su imagen corporal, de manera particular las mujeres.

La escuela como espacio de interacción propicia vínculos afectivos que favorecen la consolidación de la autoimagen (Gómez, et al., 2016). Sin embargo, se ha evidenciado que los docentes de primaria al referirse a la autoimagen de sus estudiantes suelen expresar ideas de acuerdo al grado de cercanía con sus estudiantes (Alvarez, 1991), lo cual evidencia inestabilidad en las apreciaciones de los profesores respecto a la autoimagen de sus estudiantes. De acuerdo a estas reflexiones la labor docente, además de la responsabilidad profesional, implica un alto compromiso y desarrollo de habilidades sociales y empáticas que le permitan conocer y asumir las diferentes personalidades de sus estudiantes y los estados de ánimo que se presentan en el contexto escolar (Buitrago \& Herrera 2013). Todo ello, para aportar a la configuración de la autoimagen del alumnado.

Se requiere prestar especial atención a las percepciones negativas de las personas y su incidencia en la autoimagen, dado que en muchas ocasiones se difuminan tras impresión positiva y pueden afectar la aceptación personal y la representación mental de sí mismo (Odebode, 2020). La autoimagen tiene que ver con un modelo de apreciación aceptable que integra la motivación de sí mismo, el conocimiento y el papel que juegan los valores y sentimientos en cada persona. Así mismo, se pueden señalar tres dimensiones que, como es lógico, inciden de manera positiva o negativa en la autoimagen: las transiciones escolares, las interacciones entre pares y la relación con la familia (Roncancio \& Mattos, 2019), por esta razón, es fundamental el papel que juegan los padres de familia y los maestros.

Por su parte, el autoconcepto se refiere a la noción que tienen los sujetos respecto a las características y adjetivos que usan para definirse a sí mismos, para distinguirse de los demás (Gaeta \& Cavazos, 2017). Integra las cualidades, emociones, capacidades y habilidades, incluso la aceptabilidad social, para lograr la configuración que resalta las diferencias interindividuales y permiten reafirmar la percepción de sí mismo en un contexto social determinado, para poder desenvolverse y asumir la cotidianidad (Alegría et al., 2016). Por lo tanto, se puede señalar la importancia de fortalecer desde la infancia la concepción del propio concepto, puesto que para lograrlo se debe integrar y articular un amplio conjunto de percepciones, experiencias, imágenes, reflexiones y pensamientos respecto a sus condiciones físicas, emocionales y psicológicas. Es decir, el autoconcepto tiene una disposición multidimensional que de manera particular le permite ser constante y a la vez flexible (Ibarra \& Jacobo, 2016).

Desde otra perspectiva, Palomera et al. (2019) afirman que al trabajar la toma responsable de decisiones individuales y sociales, se deben tener en cuenta las acciones éticas como parte del autoconocimiento, las cuales implican el desarrollo de ciertas habilidades como tener conciencia de uno mismo, trabajar la autorregulación para exteriorizar las emociones de manera adecuada, tener conciencia social teniendo 
empatía por los demás, y las relaciones sociales sanas para prevenir conflictos, y buscar soluciones apropiadas. Pero, además, al fortalecer el autoconcepto en el contexto escolar, se le brinda al alumnado la oportunidad de reconocer las destrezas y limitaciones para afrontar el aprendizaje (Miravet et al., 2011).

Por último, la autoestima está asociada al gusto, valoración y aceptación de sí mismo, es decir, se orienta a tener una imagen positiva de sí y a estar satisfecho, además de mantener buenas relaciones consigo mismo. Por esta razón, la autoestima puede llegar a ser positiva y propiciar confianza, autoaceptación, optimismo y la no preocupación excesiva frente a lo que piensan otros, por nombrar algunas de ellas. Pero también, en ocasiones llega a ser negativa y generar falta de confianza, inseguridad, exceso de preocupación por los pensamientos de otras personas e incluso pesimismo, entre muchas otras (López, 2017). En conclusión, la autoimagen se refiere a la manera en que las personas se ven a sí mismas, a lo que se suma el autoconcepto que configura cada persona y que permite establecer la autoestima, ya que en esencia esta se refiere a cómo se siente cada quien consigo mismo (López, 2017).

\section{Autoimagen, Autoconcepto y Autoestima del Profesorado}

Es innegable que los maestros se establecen como referente positivo o negativo de sus alumnos y que el éxito de sus procesos y la calidad de su labor está asociada a su nivel de desarrollo integral, del cual, la dimensión emocional es indispensable, ya que, además de propiciar estabilidad y bienestar, aporta una serie de habilidades y recursos pedagógicos, didácticos y educativos. Tal y como lo ha establecido la IE, el ámbito intrapersonal es fundamental porque aporta a todas las dimensiones del ser; aspectos que son retomados por modelos de evaluación y análisis de la IE.

Por ejemplo, desde el modelo socioemocional de Bar-On (1997, 2000, 2006), lo intrapersonal se suma y vincula al ámbito interpersonal, a la adaptabilidad, al manejo del estrés, a la impresión positiva y al estado general de ánimo. Desde el modelo de habilidades socioeomocionales de Mayer y Salovey (Brackett \& Salovey, 2006; Mayer \& Salovey, 1997, 2007; Salovey \& Mayer, 1990) la esfera de lo intrapersonal se relaciona con la percepción, valoración y expresión de la emoción; las emociones como facilitadoras del pensamiento; la habilidad para comprender la emoción a partir del conocimiento emocional y; la regulación reflexiva de las emociones para promover el crecimiento intelectual y emocional.

Por consiguiente, la autoimagen, el autoconcepto y la autoestima del profesorado son fundamentales y no deberían asumirse tan solo como aspectos naturales que de una u otra manera se desarrollan por sí mismos, sino que requieren estudiarse, analizarse y trabajarse desde los procesos de formación del profesorado y desde las políticas y los sistemas educativos. Se puede señalar que la autoimagen del docente se configura a partir de su propia interioridad y de su relación con el contexto, es decir, por factores intrínsecos y extrínsecos (Belousov et al., 2015). Es importante 
reconocer que no todos los maestros llegan a la docencia por vocación o convicción, o incluso con la certeza de su elección profesional, además, los conflictos, desafíos y circunstancias tanto personales como laborales pueden generar que su autoimagen no siempre se haya configurado de manera adecuada, positiva o edificante.

El autoconcepto, es la suma de creencias del individuo sobre sus cualidades personales, es decir, lo que sabe de sí mismo al igual que lo que cree que sabe, como consecuencia, este concepto se configura, modifica y resignifica a lo largo de la vida (López, 2017). Por esta razón, es más adecuado ilustrar el autoconcepto desde sus magnitudes (Gaeta \& Cavazos, 2017), por ejemplo, desde lo corporal y lo académico. Es relevante resaltar que el autoconcepto se nutre de vivencias adquiridas en diferentes contextos (Ibarra \& Jacobo, 2016). Llegados a este punto, se puede afirmar que la relevancia del fortalecimiento de la autoimagen y el autoconcepto radica en que se verán reflejados en los docentes a través de su autoconfianza y un alto nivel de autoestima.

El autoconcepto, que aporta a la individualidad y unicidad del maestro, se va enriqueciendo y construyendo a partir de experiencias, conceptos, éxitos y fracasos. En esta dirección, desde la perspectiva de Lohbeck et al. (2018) son seis los aspectos que integran el autoconcepto del maestro: las habilidades pedagógicas, los conocimientos del contenido de la asignatura, la consultoría, la innovación, el uso de medios y el diagnóstico, los cuales están relacionados con las emociones. Debido a esto, se puede señalar que un contexto escolar que propicie las interacciones, los vínculos y la autonomía, es primordial para el desarrollo de la profesión docente y para aportar a su bienestar, satisfacción laboral y autoconcepto. Por esta razón, se evidencia un mejor desempeńo del profesorado en cuanto a la autoeficacia docente y la identificación con la escuela cuando se integra en la cotidianidad el diálogo reflexivo respecto a la enseñanza, así como una comunicación respetuosa y asertiva.

Es fundamental estar atento a los aspectos profesionales y personales del profesorado (McInerney et al., 2018). Al respecto, Ross et al. (2012) enfatizan en la importancia de implementar programas para fortalecer el comportamiento positivo, hacer uso de estrategias orientadas al afrontamiento (Parker et al., 2012) y para el control del desempeño y la autorreflexión (autorregulación cognitiva) (Mattern \& Bauer, 2014), como estrategia para evitar el cansancio y agotamiento emocional. A esto se suma que, en el profesorado, el autoconcepto está incidido de manera importante por la percepción que tienen de sí mismos respecto a su desempeño comunicativo y, como es lógico, cuando esta valoración es positiva se relaciona con la satisfacción y bienestar, sin embargo cuando el autoconcepto es bajo se hace necesario fortalecer el bienestar psicológico (Glotova \& Wilhelm, 2014).

Por su parte, la autoestima se puede definir como una percepción mental que el sujeto establece sobre sí mismo y que, de alguna manera, le permite sentirse y desarrollarse en un determinado contexto. Esta configuración mental cohabita con 
las demás ideas mentales (García et al., 2019). Sumado a ello, su carga emocional y afectiva le otorga una representación personal, por lo tanto, se puede complementar que la autoestima también hace referencia al grado de confianza, aceptabilidad y valor afectivo que una persona se tiene e implica la relación que las personas logran entre sus virtudes, defectos, potencial y limitaciones (Asakereh \& Yousofi, 2018). Los docentes que cuentan con mayores habilidades emocionales suelen tener mayor empatía con sus estudiantes y logran una importante interacción entre emociones, conocimiento y comportamiento (Bisquerra, 2007, 2009).

Se puede afirmar que la autoestima y el autocontrol son fundamentales para todas las personas, pero aún más, para el profesorado, ya que la propia aceptación como maestro es fundamental para el bienestar personal y del alumnado, en gran medida, porque representa un aporte para la configuración de la interioridad de los discípulos, por el apoyo social y por la ayuda que el maestro puede aportar para abordar los problemas de externalización, sobre todo en los niños (Zhang et al., 2021). Al respecto, se podría comentar que, al analizar un grupo de maestros en formación se evidenció que aquellos que contaban con mayor autoestima asumían estilos positivos de afrontamiento frente al estrés, mientras que quienes tenían bajos niveles asumían comportamientos de evitación (Onen \& Ulosoy, 2015). Por esta razón, quienes cuentan con mejor autoestima suelen resolver de manera positiva las situaciones difíciles y los problemas (D'Zurilla et al., 2003; Hamarta, 2009).

La autoestima funciona como predictor de la felicidad (Hwang et al., 2015), la cual es vital para el profesorado porque de ella depende, en gran medida, el aprendizaje, las interacciones, los vínculos positivos, la autonomía, la empatía, la sensibilidad, la intuición social, el altruismo, la creatividad, las posturas críticas y, de alguna manera, el fomento del pensamiento. Además, Phillips (2021) señala la importancia y relevancia del ámbito espiritual para la autoestima. Es por ello que, tal y como se ha venido planteando, lograr una autoestima adecuada en el profesorado se debe asumir como una prioridad para los sistemas educativos, las universidades e instituciones que tienen a su cargo la formación del profesorado.

Para concluir, es fundamental enfatizar en la identidad profesional docente, la cual se nutre y a la vez aporta a la autoimagen, el autoconcepto y la autoestima. Stenberg y Maaranen (2021) enfatizan en la importancia de los primeros ańos de experiencia laboral en el proceso de configuración de la identidad docente, en gran medida, porque se confrontan las perspectivas personales con las relacionadas a la enseñanza; en particular con aspectos pedagógicos y didácticos, la gestión del aula, los vínculos y relación con el alumnado y la motivación y evaluación. Para Hanna et al. (2020), la identidad docente está constituida de manera preponderante por cuatro aspectos fundamentales: la autoimagen, la motivación, la percepción de la tarea y la autoeficacia.

De otro lado, Richter et al. (2021) evidenciaron, al estudiar a un grupo de 
formadores del profesorado, que estos se podían clasificar en dos grupos asociados a su identidad profesional: transmisores o facilitadores, siendo estos últimos los que utilizaban mayores recursos emocionales para la enseńanza, apostaban de manera positiva por el aprendizaje y presentaban mayor sensación de autoeficacia y satisfacción laboral. La identidad profesional docente es heterogénea, dinámica y se establece a partir de experiencias emocionales intrapersonales e interpersonales, muchas de ellas en el aula de clase y en distintos contextos (Buitrago-Bonilla \& Cárdenas-Soler, 2017).

El último aspecto por resaltar en esta reflexión respecto a la importancia de la autoimagen, el autoconcepto y la autoestima del profesorado, se orienta a señalar que la labor docente implica el riesgo de llegar al agotamiento e incluso derivar en problemas de salud tanto física como mental que suelen ser gatillados, en muchas ocasiones, por el estrés y los conflictos que se presentan en el ámbito laboral (Phillips, 2021). Por esta razón, se ha venido prestando mayor atención al burnout, el cual se conoce como el síndrome de desgaste profesional y tiene un impacto significativo en el ámbito físico y psicológico del profesorado (Bakker et al., 2007; Durán et al., 2006; Extremera et al., 2003). Por consiguiente, son muy importantes los programas que enfatizan en el manejo de los eventos adversos desde el mismo quehacer docente y el apoyo profesional, enfocados en muchas ocasiones en el autocontrol y la autoestima como mediadores de los efectos que acarrea el estrés laboral sobre el ajuste emocional del profesorado (Extremera, et al., 2003).

En cualquier caso, los docentes experimentan una gran variedad de emociones tanto positivas como negativas durante el desarrollo de sus clases, ellas a su vez juegan un papel primordial en la motivación de los estudiantes ya que influyen en su modo de pensar y actuar en clase (Extremera \& Fernandez, 2015). Por esta razón se puede resaltar, una vez más, que los docentes son un modelo fundamental para los alumnos y que sus habilidades socioemocionales median sobre el manejo de la convivencia y el clima escolar (Gutiérrez-Torres \& Buitrago-Velandia, 2019), lo cual se logra al propiciar una complementariedad entre el ámbito intrapersonal e interpersonal (Buitrago et al., 2017). Los docentes que pueden llegar a la mente, corazón y alma de sus discípulos con sus cualidades personales, las cuales se apoyan en su identidad profesional y por consiguiente en su nivel de aceptación, generan cambios positivos en sus estudiantes (Buitrago-Bonilla \& Cárdenas-Soler, 2017).

Por lo tanto, el perfil del profesorado en cuanto a su autoimagen, autoconcepto y autoestima es determinante para el desarrollo de las habilidades del estudiantado. Trabajar el cultivo y adquisición de habilidades socioemocionales en el profesorado, desde su formación inicial y de manera posterior con la formación continuada y posgraduada, es una posibilidad, una alternativa y una apuesta que debe ser tenida en cuenta (Bisquerra et al., 2015; Buitrago, 2012; Buitrago et al., 2017; Dalai-Lama \& Ekman, 2009; Darder, 2017; Extremera \& Fernández-Berrocal, 2003; Extremera et 
al., 2019; Fernández-Berrocal \& Extemera, 2002; Goleman, 1996; Greenberg, 2003; Herrera \& Buitrago, 2019; Herrera et al., 2015; Herrera et al., 2016; Lantieri, 2010; Palomera, 2017; Palomera et al., 2006; Palomera et al., 2008; Palomera et al., 2019; Petrides et al., 2006; Royo, 2017; Sala \& Marzo, 2017; Sánchez, 2019).

\section{Reflexión Final}

Es fundamental que la escuela integre el desarrollo de habilidades socioemocionales en sus procesos; que la formación inicial, continuada y posgraduada del profesorado aborde la IE como alternativa y posibilidad pedagógica y didáctica; que se le de atención al bienestar y a la humanidad del profesorado; y que se asuma la autoimagen, el autoconcepto y la autoestima como tres perspectivas diferentes, aunque complementarias, que son indispensables, esenciales para la consolidación del ámbito intrapersonal y que además son susceptibles de desarrollo.

\section{Referencias}

Acòn, S., Ballard, S., Montero, F., \& Romero, M. (2019). Efectos psicosociales del uso de Facebook en la autoimagen y habilidades sociales en estudiantes universitarios. Pro Veritatem, 5(5), 52-67. https://revistas.uia.ac.cr/index.php/proveritatem/ article/view/109/183

Adolphs, R. (2005). Could a robot have emotions? Theoretical perspectives from social cognitive neuroscience. En J. M. Fellows, \& M. A. Arbit (Eds.), Who needs emotions? The brain meets the robot (pp. 9-25). Oxford University Press.

Agulló, M., Filella, G., Soldevila, A., \& Ribes, R. (2011). Evaluación de educación emocional en el ciclo medio de Educación Primaria. Revista de Educación, 354, 765-783. https://doi.org/10.4438/1988-592X-RE-2011-354-020

Alegría, A., Guerra, E., \& Carranza, R. (2016). Autoconcepto y depresión en estudiantes universitarios. Apuntes Universitarios. Revista de Investigación, 6(2), 53-68. https://www.redalyc.org/pdf/4676/467647511004.pdf

Alvarez, J. (1991). Sagacidad perceptiva de los profesores de enseñanza primaria. Tabanque: Revista pedagógica, (7), 53-74. https://dialnet.unirioja.es/servlet/ articulo? codigo $=2254669$

Amador, C., \& Treglown, L. (2020). What makes an engaged employee? A facet-level approach to trait emotional intelligence as a predictor of employee engagement. Personality and Individual Differences, 159, Artículo número 109892. https://doi. org/10.1016/j.paid.2020.109892

Ariza-Hernández, M. L. (2017). Influencia de la inteligencia emocional y los afectos en la relación maestro-alumno, en el rendimiento académico de estudiantes de educación superior. Educación y Educadores, 20(2), 193-210. https://doi. 
org/10.5294/edu.2017.20.2.2

Asakereh, A., \& Yousofi, N. (2018). Reflective Thinking, Self-Efficacy, Self-Esteem and Academic Achievement of Iranian EFL Students in Higher Education: Is there a Relationship? International Journal of Educational Psychology, 7(1), 68-89. https://doi.org/10.17583/ijep.2018.2896

Bakker, A. B., Westman, M., \& Schaufeli, W. B. (2007). Crossover of burnout: An experimental design. European Journal of Work and Organizational Psychology, 16(2), 220-239. https://doi.org/10.1080/13594320701218288

Bar-On, R. (1997). Bar-On Emotional Quotient Inventory: Technical Manual. Multi Health System Inc.

Bar-On, R. (2000). Emotional and social intelligence: insights from the emotional quotient inventory. En R. Bar-On, \& J. Parker (Eds.), Handbook of emotional intelligence (pp. 363-388). Jossey-Bass.

Bar-On, R. (2006). The Bar-On model of Emotional-Social Intelligence (ESI). Psicothema, 18(Suppl.), 13-25.

Barrientos, A., Sánchez, R., \& Arigita, A. (2019). Formación emocional del profesorado y gestión del clima de su aula. Praxis \& Saber, 10(24), 119-141. https://doi.org/10.19053/22160159.v10.n25.2019.9894

Belousov, K., Erofeeva, E., Erofeeva, T., Leshchenko, Y., \& Zelyanskaya, N. (2015). University Teachers of Linguistics and Self-Image of Their Profession. Procedia - Social and Behavioral Sciences, 214, 667-676. https://www.researchgate.net/ publication/287158049_University_Teachers_of_Linguistics_and_Self-Image_ of_Their_Profession

Bisquerra, R. (2007). Educación emocional y bienestar. Praxis.

Bisquerra, R. (2009). Psicopedagogía de las Emociones. Síntesis.

Bisquerra, R., Peréz, J. C., \& García, E. (2015). Inteligencia Emocional en educación. Síntesis.

Brackett, M., Mayer, J., \& Warner, R. (2004). Emotional intelligence and its relation to everyday behavior. Personality and Individual Differences, 36, 1387-1402. https://doi.org/10.1016/S0191-8869(03)00236-8

Brackett, M., \& Salovey, P. (2006). Measuring emotional intelligence with the Mayer-Salovery-Caruso Emotional Intelligence Test (MSCEIT). Psicothema, 18(Suppl.), 34-41.

Buitrago, R. E. (2012). Contexto escolar e inteligencia emocional en instituciones educativas públicas del ámbito ruraly urbano del departamento de Boyacá (Colombia) [Tesis doctoral, Universidad de Granada]. http://hera.ugr.es/tesisugr/20956575. 
pdf

Buitrago, R. E., Ávila, A. K., \& Cárdenas, R. N. (2017). El sentido y el significado atribuido a las emociones por el profesorado en formación de la Universidad Pedagógica y Tecnológica de Colombia. Contextos Educativos, 20, 77-93. http:// doi.org/10.18172/con.2998

Buitrago-Bonilla, R. E., \& Cárdenas-Soler, R. N. (2017). Emociones e identidad profesional docente: relaciones e incidencia. Praxis \& Saber, 8(17), 225-247. https://doi.org/10.19053/22160159.v8.n17.2018.7208

Buitrago, R. E., \& Herrera, L. (2013). Matricular las emociones en la escuela, una necesidad educativa y social. Praxis \& Saber, 8(4), 87-108. https://revistas.uptc. edu.co/index.php/praxis_saber/article/view/2653/2468

Buitrago, R. E., Herrera, L., \& Cárdenas, R. N. (2019). Coeficiente emocional en niños y adolescentes de Boyacá, Colombia. Estudio comparativo. Praxis \& Saber, 10(24), 45-68. https://doi.org/10.19053/22160159.v10.n25.2019.10002

Cañas, D. C., \& Hernández, J. (2019). Comunicación asertiva en profesores: diagnóstico y propuesta educativa. Praxis \& Saber, 10(24), 143-165. https://doi. org/10.19053/22160159.v10.n25.2019.8936

Cherniss, C., Extein, M., Goleman, D., \& Weissberg, R. (2006). Emotional Intelligence: What Does the Research Really Indicate? Educational Psychologist, 41(4), 239-245. https://doi.org/10.1207/s15326985ep4104_4

Dalai-Lama, \& Ekman, P. (2009). Sabiduría Emocional. Kairós.

Darder, P. (2017). Emociones y educación, una integración necesaria. En P. Darder (Coord.), La Formación Emociona del Profesorado (pp. 15-30). Octaedro.

Davidson, R. J., Jackson, D. C., \& Kalin, N. H. (2000). Emotions, Plasticity, Context and Regulation: Perspectives from Affective Neuroscience. Psychological Bulletin, 126(6), 890-906. https://doi.org/10.1037/0033-2909.126.6.890

Durán, A., Extremera, N., Rey, L., Fernández-Berrocal, P., \& Montalbán F. M. (2006). Predicting academic burnout and engagement in educational settings: Assessing the incremental validity of perceived emotional intelligence beyond perceived stress and general self-efficacy. Psicothema, 18(Suppl.), 158-164. http:// www.psicothema.com/psicothema.asp? id=3292

D’Zurilla, T. J., Chang, E. C., \& Sanna, L. J. (2003). Self-esteem and social problem solving as predictors of aggression in college students. Journal of Social and Clinical Psychology, 22(4), 424-440. https://doi.org/10.1521/jscp.22.4.424.22897

Ekman, P. (2003). La universalidad de las emociones. En D. Goleman (Ed.), Emociones Destructivas, cómo entenderlas y superarlas (pp. 71-93). Editorial Kairós. 
Extremera, N., \& Fernández -Berrocal, P. (2003). La inteligencia emocional en el contextoeducativo: hallazgos científicos de sus efectos en elaula. RevistadeEducación, 332, 97-116. https://dialnet.unirioja.es/servlet/articulo?codigo=776719

Extremera, N., Fernández-Berrocal, P., \& Duran, A. (2003). Inteligencia Emocional y burnout en profesores. Encuentros en Psicología Social, 1, 260-265. https:// emotional.intelligence.uma.es/documentos/PDF5burnout_en_profesores.pdf

Extremera, N., \& Fernández-Berrocal, P. (2015). Inteligencia Emocional y Educación. Editorial Grupo 5.

Extremera, N., Mérida-López, S., Sánchez-Álvarez, N., Quintana-Orts, C., \& Rey, L. (2019). Un amigo es un tesoro: inteligencia emocional, apoyo social organizacional y engagement docente. Praxis \& Saber, 10(24), 69-92. https://doi. org/10.19053/22160159.v10.n25.2019.10003

Engels, M. C., Spilt, J., Denies, K., \& Verschueren, K. (2021). The role of affective teacher-student relationships in adolescents' school engagement and achievement trajectories. Learning and Instruction, 75, Artículo número 101485. https://doi. org/10.1016/j.learninstruc.2021.101485

Eren, E., Ergun, E., \& Altintas, O. (2009). The Relationship Between School Success and the Emotional Intelligence of Primary School Headmasters and Teachers. Journal of American Academy of Business, Cambridge, 15(1), 224-228 224. http:// www.jaabc.com/jaabcv15n1 preview.html

Fernández-Berrocal, P., \& Extemera, N. (2002.). La Inteligencia Emocional como una habilidad esencial en la Escuela. Revista Iberoamericana de Educación, 29, 1-6. https://emotional.intelligence.uma.es/documentos/PDF8habilidad_esencial_ en_la_escuela.pdf

Gaeta, M., \& Cavazos, J. (2017). Autoconcepto físico y académico en nińos de contextos marginados en México. Revista electrónica de investigación educativa, 19(2), 114-124. https://doi.org/10.24320/redie.2017.19.2.604

García, R., Canuto, I., \& Cebrián, A. (2019). Alta capacidad y género: la autoestima como factor influyente en las diferencias entre sexos entre sexos. Contextos Educativos, (24), 77-93. https://doi.org/10.18172/con.3934

Gil-Olarte, M., Palomera, R., \& Brackett, M. (2006). Relating emotional intelligence to social competence and academic achievement in high school students. Psicothema, 18(Suppl.), 118-123. https://www.redalyc.org/pdf/727/72709518. pdf

Glotova, G., \& Wilhelm, A. (2014). Teacher's self-concept and self-esteem in pedagogical communication. Procedia - Social and Behavioral Sciences, 132, 509514. 
Goleman, D. (1996). Inteligencia emuocional. Kairos.

Gómez, M., Conejeros-Solar, M., Sandoval, K., \& Armijo, S. (2016). Doble excepcionalidad: análisis exploratorio de experiencias y autoimagen en estudiantes chilenos. Revista de Psicología, 34(1), 5-37. http://dx.doi.org/10.18800/ psico.201601.001

Gómez-Mármol, A., Sánchez-Alcaraz, B. J., Molina-Saorín, J., \& Bazaco, M. J. (2017). Violencia Escolar y Autoimagen en Adolescentes de la Región de Murcia (España). Revista Internacional de Medicina y Ciencias de la Actividad Física y del Deporte, 17(68), 677-692. https://www.redalyc.org/pdf/542/54254647007.pdf

Greenberg, M. T. (2003). La educación del corazón. En D. Goleman (Ed.), Emociones Destructivas, cómo entenderlas y superarlas (pp. 146-158). Kairós.

Greenberg, M., Weissberg, R., O’Brien, M., Zins, J., Fredericks, L., Resnik, H., \& Elias, M. (2003). Enhancing school-based prevention and youth development through coordinated social and emotional learning. American Psychologist, 58, 466-474. https://doi.org/10.1037/0003-066X.58.6-7.466

Gutiérrez-Torres, A. M., \& Buitrago-Velandia, S. J. (2019). Las habilidades socioemocionales de los docentes, herramientas de paz en la escuela. Praxis \& Saber, 10(24), 167-192. https://doi.org/10.19053/22160159.v10.n25.2019.9819

Hamarta, E. (2009). A prediction of self-esteem and life satisfaction by social problem solving. Social Behavior and Personality, 37(1), 73-82. https://doi.org/10.2224/ sbp.2009.37.1.73

Hanna, F., Oostdam, R., Severiens, S. E., \& Zijlstra, B. J. (2020). Assessing the professional identity of primary student teachers: Design and validation of the Teacher Identity Measurement Scale. Studies in Educational Evaluation, 64, Artículo número 100822. https://doi.org/10.1016/j.stueduc.2019.100822

Herrera, L., \& Buitrago, R. E. (2019). Emociones en la educación en Colombia, algunas reflexiones [Editorial]. Praxis \& Saber, 10(24), 9-22. https://www.redalyc. org/journal/4772/477266187001/477266187001.pdf

Herrera, L., Buitrago, R. E., \& Ávila, A. K. (2016). Empathy in future teachers of the Pedagogical and Technological University of Colombia. New Approaches in Educational Research, 5(1), 30-37. https://doi.org/10.7821/naer.2016.1.136

Herrera, L., Buitrago-Bonilla, R. E., \& Cepero, S. (2017). Emotional Intelligence in Colombian Primary School Children. Location and Gender. Universitas Psychologica, 16(3), 1-10. https://doi.org/10.11144/Javeriana.upsy16-3.eips

Herrera, L., Buitrago, R. E., Lorenzo, O., \& Badea, M. (2015). Socio-Emotional Intelligence in Colombian Children of Primary Education. An analysis in rural and urban settings. Procedia - Social and Behavioral Sciences, 203, 4-10. https:// 
doi.org/10.1016/j.sbspro.2015.08.251

Herrera, L., Buitrago, R. E., Lorenzo, O., \& Perandones, T. M. (2015). Psicología Positiva e Inteligencia Emocional en Educación. DEDiCA, Revista de Educação e Humanidades, 8, 139-153. https://hum742.ugr.es/media/grupos/HUM742/ descargas/DEDiCA\%20COMPLETA\%20ELECTRONICA__.pdf\#page=141

Hill, N. L. (2013). Self Concept: Self-Image, Ideal Self, and Self-Esteem. http:// practicewisdom.blogspot.com.es/2013/06/self-concept-self-image-ideal-self-and. html

Hubel, G. S., Davies, F., Goodrum, N. M., Schmarder, K. M., Schnake, K., \& Moreland, A. D. (2020). Adverse childhood experiences among early care and education teachers: Prevalence and associations with observed quality of classroom social and emotional climate. Children and Youth Services Review, 111, Artículo número 104877. https://doi.org/10.1016/j.childyouth.2020.104877

Hwang, H., Kang, H., Tak, J., \& Lee, S. (2015). Impact of Self-esteem and Gratitude Disposition on Happiness in Pre-service Early Childhood Teacher. Procedia - Social and Behavioral Sciences, 174, 3447-3453. https://doi.org/10.1016/j. sbspro.2015.01.1017

Ibarra, E., \& Jacobo, H. (2016). La evolución del autoconcepto académico en adolescentes. Revista mexicana de investigación educativa, 21(68), 45-70. http:// www.scielo.org.mx/pdf/rmie/v21 n68/1405-6666-rmie-21-68-00045.pdf

Lantieri, L. (2010). Las emociones van a la escuela. National Geographic, edición especial, 66-73. https://www.cbtis59.edu.mx/Descargables/Las-Emociones-van-ala-escuela.pdf

López, M. (2017). Análisis del orden en el que el autoconcepto, la autoestima y la autoimagen deberían aparecer en el proceso de maduración personal para alcanzar el bienestar emocional. Revista INFAD de Psicología, 1(2), 257-264. https://doi. org/10.17060/ijodaep.2017.n2.v1.1126

Lohbeck, A., Hagenauer, G., \& Frenzel, A. C. (2018). Teachers' self-concepts and emotions: Conceptualization and relations. Teaching and Teacher Education, 70, 111-120. https://doi.org/10.1016/j.tate.2017.11.001

Maganto, C., \& Maganto, J. M. (2010). Cómo potenciar las emociones positivas y afrontar las negativas. Pirámide.

Masschelein, J., \& Simons, M. (2014). Defensa de la escuela: una cuestión pública. Miño y Dávila.

Mattern, J., \& Bauer, J. (2014). Does teachers' cognitive self-regulation increase their occupational well-being? The structure and role of self-regulation in the teaching context. Teaching and Teacher Education, 43, 58-68. https://doi.org/ 10.1016/j. 
tate.2014.05.004

Mayer, J. D., \& Salovey P. (1997). ¿What is emotional intelligence? En P. Salovey, \& D. Sluyter (Eds.), Emotional development and emotional intelligence: implications for educators (pp. 3-34). Basic Books.

Mayer, J. D., \& Salovey, P. (2007). Mayer-Salover y-Caruso emotional intelligence test. Multi-Health Systems Incorporated.

McInerney, D. M., Korpershoek, H., Wang, H., \& Morin, A. J. S. (2018). Teachers' occupational attributes and their psychological wellbeing, job satisfaction, occupational self-concept and quitting intentions. Teaching and Teacher Education, 71, 145-158. https://doi.org/10.1016/j.tate.2017.12.020

Mestre, J., Gutiérrez-Trigo, J., Guerrero, C., \& Guil, R. (2017). Gestión de emociones en el día a día. Ediciones Pirámide.

Miravet, L., Flores, M., \& Durán, D. (2011). Efectos sobre la mejora de las competencias lingüísticas y la autoimagen lectora a través de un programa de tutoría entre iguales. Revista de Investigación en Educación, 9(2), 209-222. https:// core.ac.uk/download/pdf/61401882.pdf

Odebode, A. A. (2020) Self-image of in-School Adolescents in Offa Local Government Area of Kwara State, Nigeria: Implications for Counselling Practices. International Journal of Instruction, 13(1), 532-534. https://doi.org/10.29333/iji.2020.13134a

Onen, A. S., \& Ulosoy, F. M. (2015). Investigating of The Relationship between PreService Teachers' Self-Esteem and Stress Coping Attitudes. Procedia - Social and Behavioral Sciences 186, 613-617. https://doi.org/10.1016/j.sbspro.2015.04.194

Palomera, R. (2017). Psicología Positiva en la escuela: un cambio con raíces profundas. Papeles del Psicólogo, 38(1), 66-71. https://doi.org/10.23923/pap. psicol2017.2823

Palomera, R., Briones, E., \& Gómez-Linares, A. (2019). Formación en valores y competencias socio-emocionales para docentes tras una década de innovación. Praxis \& Saber, 10(24), 93-117. https://doi. org/10.19053/22160159.v10. n25.2019.9116

Palomera, R., Fernández-Berrocal, P., \& Brackett, M. A. (2008). La inteligencia emocional como una competencia básica en la formación inicial de los docentes: algunas evidencias. Revista electrónica de investigación Psicoeducativa, 15(6), 437-454. https://emotional.intelligence.uma.es/documentos/PDF67preservice_ teacher_training.pdf

Palomera, R., Gil-Olarte, P., \& Brackett, M. A. (2006). ¿Se perciben con inteligencia emocional los docentes? Posibles consecuencias sobre la calidad educativa. Revista de Educación, 341, 687-703. https://dialnet.unirioja.es/servlet/ 
articulo?codigo $=2165292$

Parker, P. D., Martin, A. J., Colmar, S., \& Liem, G. A. (2012). Teachers' workplace wellbeing: Exploring a process model of goal orientation, coping behavior, engagement, and burnout. Teaching and Teacher Education, 28, 503-513. https:// doi.org/10.1016/j.tate.2012.01.001

Pérez, N., \& Filella, G. (2019). Educación emocional para el desarrollo de competencias emocionales en niños y adolescentes. Praxis \& Saber, 10(24), 2344. https://doi.org/10.19053/22160159.v10.n25.2019.8941

Petrides, K. V., Sangareau, Y., Furnham, A., \& Frederickson, N. (2006). Trait Emotional Intelligence and Children's Peer Relations at School. Social Development, 15(3), 537-547. https://doi.org/10.1111/j.1467-9507.2006.00355.x

Phillips, R. (2021). Teachers' faith, identity processes and resilience: a qualitative approach. British Journal of Religious Education. Publicación anticipada en línea. https://doi.org/10.1080/01416200.2021.1891860

Porras, L. T., Buitrago, R. E., \& Gutiérrez, A. M. (2020). Comunicación Emocional No Verbal en la Infancia, Análisis en el Aula desde la Metodología Observacional. Know and Share Psychology, 1(4), 211-228. http://dx.doi.org/10.25115/kasp. v1i4.4343

Rendón, M. A. (2019). Competencias Socioemocionales de maestros en formación y egresados de programas de educación. Praxis \& Saber, 10(24), 243-270. https:// doi.org/10.19053/22160159.v10.n25.2019.10004

Richter, E., Brunner, M., \& Richter, D. (2021). Teacher educators' task perception and its relationship to professional identity and teaching practice. Teaching and Teacher Education, 101, Artículo número 103303. https://doi.org/10.1016/j. tate.2021.103303

Roncancio, M., \& Mattos, E. (2019). Self-Imaging: Beyond Children's and Adolescents'. Estudios de Psicología, 40(1), 157-185. https://doi.org/10.1080/021 09395.2018 .1565028

Ross, S. W., Romer, N., \& Horner, R. H. (2012). Teacher well-being and the implementation of schoolwide positive behavior interventions and supports. Journal of Positive Behavior Interventions, 14, 118-128. https://doi.org/10.1177/ 1098300711413820.

Royo, M. (2017). Nuevas Perspectivas en la Formación Inicial del Profesorado. En P. Darder (Coord.), a formación emocional del profesorado (pp. 169-190). Octaedro.

Sala, J., \& Marzo, L. (2017). Emociones y procesos educativos: los estilos educativos y las emociones. En P. Darder (Coord.), La formación emocional del profesorado (pp. 121-144). Octaedro. 
Salovey, P., \& Mayer, J. (1990). Emotional intelligence. Imagination, cognition and personality, 9(3), 185-211. https://doi.org/10.2190\%2FDUGG-P24E-52WK6CDG

Sánchez, L. (2019). La formación inicial docente en una universidad chilena: estudiando el ámbito emocional. Praxis \& Saber, 10(24), 217-242. https://doi. org/10.19053/22160159.v10.n25.2019.8931

Stenberg, K., \& Maaranen, K. (2021). A novice teachers teacher identity construction during the first year of teaching: A case study from a dialogical self perspective. Learning, Culture and Social Interaction, 28, Artículo número 100479. https:// doi.org/10.1016/j.lcsi.2020.100479

Zhang, R., Qiu, Z., Li, Y., Liu, L., \& Zhi, S. (2021). Teacher support, peer support, and externalizing problems among left-behind children in rural China: Sequential mediation by self-esteem and self-control. Children and Youth Services Review, 121, Artículo número 105824. https://doi.org/10.1016/j.childyouth.2020.105824

Zembylas, M. (2003). Emotions and Teacher Identity: a poststructural perspective. Teachers and Teaching: theory and practice, 9(3), 213-238. http://dx.doi. org/10.1080/1354060032000116611 